\title{
SYNERGETIC CONTROL FOR HVAC SYSTEM CONTROL AND VAV BOX FAULT COMPENSATION
}

\author{
RADHIA ETTOUIL $^{a, *}$, KARIM CHABIR ${ }^{a}$, DOMINIQUE SAUTER ${ }^{b}$, \\ MOHAMED NACEUR ABDELKRIM ${ }^{a}$ \\ ${ }^{a}$ National Engineering School of Gabes, Research Unit MACS 06/UR/11-12 \\ University of Gabes, Rue Omar Ibn El Khattab, Gabes 6029, Tunisia \\ e-mail: radhiaettouilegmail.com \\ ${ }^{b}$ Research Center for Automatic Control of Nancy, CRAN UMR 7039 \\ Lorraine University, 54500 Vandeouvre Les Nancy, France \\ e-mail: dominique.sauter@univ-lorraine.fr
}

\begin{abstract}
Synergetic control is proposed for heating, ventilating and air-conditioning (HVAC) system control. The synergetic controller is developed using the nonlinear model of the HVAC system. Occupancy information in each zone is required in the design of the controller which offers inherent comfort according to the occupancy in the zone. The stability of the building system using the proposed control is verified through the Lyapunov approach. It is also proved that the synergetic controller is robust to external disturbances. Then, synergetic theories are used to design a reconfigurable control for damper stuck failures in variable air volume (VAV) to recover the nominal performance. Simulations are provided to validate the effectiveness of the proposed controller for a three-zone building.
\end{abstract}

Keywords: HVAC system, synergetic control, reconfigurable control, robustness.

\section{Introduction}

Heating, ventilating and air-conditioning (HVAC) systems are widely used in buildings to provide thermal comfort and adequate indoor air quality. Certain HVAC system features, such as time-varying bilinear system dynamics, time-varying disturbances and an interacting control loop, render its control challenging. In this context, several control methods have been implemented for HVAC systems. Because of its simplicity, proportional integral derivative (PID) control is applied in many HVAC systems (Bai and Zhang, 2007). But, it is not optimal in both thermal comfort and energy consumption. In the last few years, the most commonly used control has been model predictive control (MPC), and thus several MPC strategies have been developed (Afram and Janabi-Sharifi, 2014).

A supervisory (MPC) controller was developed and implemented for residential HVAC systems (Afram and Janabi-Sharifi, 2017), which uses the weather forecast and electricity TOU price information to find an optimum

* Corresponding author set-point trajectory. In the work of Seybold et al. (2015) a control framework comprising an AFTC and reference redesign is proposed subject to actuator stuck failures. In the work of Aftab et al. (2017) an automatic HVAC control system is implemented in a low-cost embedded system which includes dynamic occupancy prediction and real-time occupancy recognition. A power shaping approach was proposed by Chinde et al. (2017) that exploits the properties of the system.

In the present work, another category of control, the so-called synergetic control, is proposed. The method of synergetic control is variable-structure control developed by Kolesnikov et al. (2000). The advantages of the synergetic approach are order reduction, insensitivity to parameter variations, global stability and disturbance suppression. The synergetic approach has been successfully implemented in the area of power electronics control (Jiang and Dougal, 2004; Zhu et al., 2017), and many strategies of synergetic control have been developed (Bouchama et al., 2016; Medjbeur et al., 2018).

Synergetic control uses the same principle as sliding 
mode control (SMC) in driving the system dynamics to converge towards the designed dynamics defined as manifolds. Moreover, the advantage of this control compared with SMC is that it suppresses the chattering phenomenon. It is a two-step approach consisting of firstly designing the desired dynamics, called the manifold and denoted by $s(x)$. The constructed manifold is a function of a variable state designed according to the desired system performance. The way to construct the manifold is detailed by Slotine and Li (1991). The second step is to synthesize a synergetic control law that governs the controlled system's trajectory convergence to the manifold. The principle of the synergetic control approach is presented by Nusawardhana et al. (2007) with a proof of its optimality. The synergetic control was used herein to provide thermal comfort in building zones.

The components of the HVAC system are prone to failures due to embedded electronics and their complexity (Darure et al., 2016). Each fault will surely impact thermal comfort in building zones as well as energy consumption if it is not compensated in time (Darure et al., 2016). In this circumstance, various fault tolerant control systems (FTC) for the HVAC system have lately gained attention. FTC is based on two cascading mechanisms: a fault detection and diagnosis (FDD) mechanism for the detection and identification of fault occurrence time and location. The other one is reconfigurable control, which deals with the design of new control according to the fault value to bring the system trajectories to the desired performance. Many methods of FDD for air-handling units (AHUs) are presented by Yu et al. (2014). Other methods have been proposed in the area of FDD (Sauter and Hamelin, 1999; Chen and Patton, 2012; Chabir et al., 2008; 2014; Qi et al., 2017).

A review of fault detection and diagnosis methods for HVAC system is presented by Kim and Katipamula (2018). Important fault scenarios that can affect the HVAC system are presented by Bengea et al. (2015) and Du et al. (2014). The most serious and current fault scenario is damper stuck. Once a damper is stuck, the control input can no longer be efficient, which requires a control reconfiguration to compensate for the effect of failed dampers. The control law is obtained through measured system states with adjustable parameters. The main approach by Sauter et al. (2015) is to develop an adaptive reconfigurable control using the fault estimated value obtained through a fault isolation filter.

In the paper, we design a reconfigurable control using the synergetic approach. The control is obtained after designing a manifold $s(x)=0$ along which the air temperature is equal to the desired value that will maintain a constant temperature in the affected zone. Synergetic reconfigurable control is synthesized by solving the first-order equation to drive the air temperature to get the manifold $s(x)=0$. Thereafter, it forces the air temperature to converge to the desired value that allows a satisfactory temperature in the event of such a fault.

This paper is organized as follows. The HVAC system and the problem formulation are presented in Section 2. In Section 3, synergetic control is first introduced and then applied to the HVAC system. Synergetic reconfigurable control is developed in Section 4. The performances of the proposed approach are assessed via a three-zone building system in Section 5. Concluding remarks are presented in Section 6.

\section{HVAC system description and thermal modelling}

2.1. HVAC system description. HVAC systems are used in commercial, residential and industrial buildings to guarantee the conditioned temperature, pressure and humidity in zones. Major components of a building HVAC system are the air handling unit (AHU) and the variable-air-volume (VAV) system. A mixture of outside air and recycled air is supplied to the AHU. The AHU increases (or decreases) the supply air temperature by heating coils (or cooling coils, respectively). The conditioned air is supplied to VAV boxes. VAV supplies air flow to the zone through the damper and each zone returns air (recycled air and exhaust air). The recycled air will be reutilized by another air circle again.

The focus of this work is on VAV boxes of the zones. In a VAV system, the flow rate of the supply air in the zone is varied through dampers. The dampers are driven by actuators. Synergetic control is used in this work to control those actuators. The HVAC system is illustrated in Fig. 1.

2.2. Building modelling. In this work we consider a building with $N$ zones of the same size. The dynamic equations are derived from the energy balance between conservation of mass and energy with a constant air specific heat coefficient.

For each zone $i$ its temperature and volume and the mass flow rate of the supply air are denoted by $T_{i}, V_{i}$ and $\dot{m}_{i}$, respectively. Let the supply air temperature be $T_{\text {air }}$

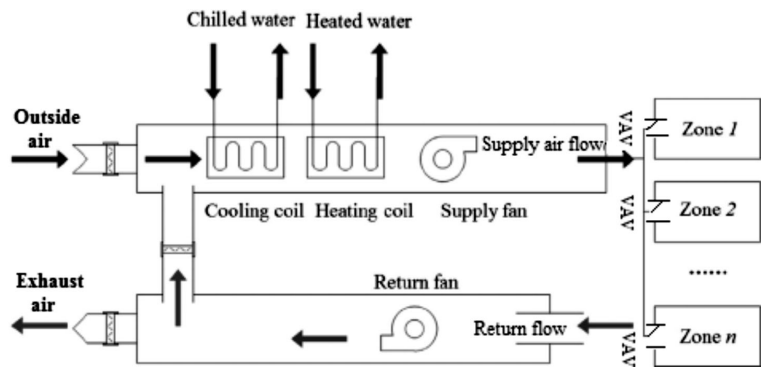

Fig. 1. Multi-zone building HVAC system. 
and the air specific heat coefficient be $c_{p}$. The thermal dynamics model of the multi-zone building is obtained using the first law of thermodynamics (Sauter et al., 2015) as follows:

$$
M_{i} c_{p} \frac{\mathrm{d} T_{i}}{\mathrm{~d} t}=Q_{\mathrm{air}, i}+Q_{\mathrm{out}_{i}}+Q_{i-1, i}+Q_{i+1, i}+g_{i}
$$

where $M_{i}=V_{i} \rho$, with $V_{i}$ being the volume of Zone $i$, $\rho$ the air density and $g_{i}$ the internal heat gain (occupancy gain), which includes heat flux as a result of occupants and electronic devices in Zone $i$. $Q_{i j}$ represents heat transfer from Zone $i$ to Zone $j, Q_{\text {out }_{i}}$ is heat transfer from Zone $i$ to the outside. $Q_{\text {air }}$ is the net heat gain due to the supply air temperature. $Q_{\text {out }_{i}}, Q_{\text {air,i }}$ and $Q_{j i}$ are given by

$$
\begin{gathered}
Q_{\mathrm{air}, i}=\dot{m}_{i} c_{p}\left(T_{\mathrm{air}}-T_{i}\right), \\
Q_{\mathrm{out}_{i}}=\frac{T_{\mathrm{out}}-T_{i}}{R_{i}}, \\
Q_{j i}=\frac{T_{j}-T_{i}}{R_{j i}},
\end{gathered}
$$

where $T_{\text {out }}$ is the outside temperature, $R_{i}$ is the thermal resistance between Zone $i$ and the outside of the building, and $R_{i j}$ is the thermal resistance between Zones $i$ and $j$ which are adjacent. Note that $R_{i j}=R_{j i}$.

The control input to the building model includes the actuators that control the opening of the dampers and the controller of the air temperature $u_{\text {air }}$. In this work, we assume that the supply air temperature is controlled by an input control $u_{\text {air }}$ and the dynamics of the air heater are assimilated to the first order transfer function:

$$
\dot{T}_{\text {air }}=-\frac{1}{\tau} T_{\text {air }}+\frac{\alpha}{\tau} u_{\text {air }}
$$

where $\tau$ is a time constant and $\alpha$ is a positive gain.

Consider a building with $N$ adjacent zones, and let the state vector be as follows:

$$
\begin{aligned}
x^{T} & =\left[\begin{array}{llll}
x_{1} & \ldots & x_{N} & x_{N+1}
\end{array}\right]^{T} \\
& =\left[\begin{array}{llll}
T_{1} & \ldots & T_{N} & T_{\text {air }}
\end{array}\right]^{T} .
\end{aligned}
$$

The control input vector is

$$
\begin{aligned}
u^{T} & =\left[\begin{array}{llll}
u_{1} & \ldots & u_{N} & u_{N+1}
\end{array}\right]^{T} \\
& =\left[\begin{array}{llll}
\dot{m}_{1} & \ldots & \dot{m}_{N} & u_{\text {air }}
\end{array}\right]^{T} .
\end{aligned}
$$

The occupancy gain is

$$
g=\left[\begin{array}{llll}
g_{1} & g_{2} & \ldots & g_{N}
\end{array}\right]^{T} .
$$

The above system can be written as the following state space form:

$$
\dot{x}=A(x)+B(x) u+E_{1} T_{\text {out }}+E_{2} g,
$$

where the matrices $A(x), B(x), E_{1}$ and $E_{2}$ are given for (1) and (5) as

$$
A(x)=\left(\begin{array}{c}
\frac{1}{M_{1} c_{p}}\left(-\frac{x_{1}}{R_{1}}+\frac{x_{2}-x_{1}}{R_{12}}\right) \\
\frac{1}{M_{2} c_{p}}\left(-\frac{x_{2}}{R_{2}}+\frac{x_{1}-x_{2}}{R_{12}}+\frac{x_{3}-x_{2}}{R_{32}}\right) \\
\vdots \\
\frac{1}{M_{N} c_{p}}\left(-\frac{x_{N}}{R_{N}}+\sum_{j=1}^{N-1} \frac{x_{j}-x_{N}}{R_{j N}}\right) \\
-\frac{1}{\tau} x_{N+1}
\end{array}\right),
$$

The performance of the control strategy highly relies on the model used to describe the HVAC system operation. The dynamics of the multi-zone building system are a nonlinear model. In the literature the above model is generally linearized around operating points. However, model linearization can degrade control performance. Therefore, direct use of the nonlinear model is more efficient for the building HVAC system. For this reason, the nonlinear model is used directly in this work.

\subsection{Problem formulation. Consider an HVAC system} described by

$$
\dot{x}=A(x)+B(x, u)+E d(t),
$$

with $E=\left[\begin{array}{ll}E_{1} & E_{2}\end{array}\right]$ and $d(t)=\left[\begin{array}{ll}T_{\text {out }} & g\end{array}\right]^{T}$.

The problem studied in this paper is to design a controller that provides the desired performance in the nominal case, and recovers the initial performance and compensates for stuck damper faults in the faulty case. 
Besides, this control has to reject the external disturbance in both cases. Under a stuck actuator, the system (7) can be presented as follows:

$$
\dot{x}=A(x)+B_{0}(x) u_{0}+B_{f}(x) u_{f}+E d(t),
$$

where $B_{0}(x)$ is the matrix associated with the safe control inputs $u_{0}$ and $B_{f}(x)$ is the matrix associated with the affected control inputs $u_{f}$. In this effort, a robust reconfigurable control has to be designed by adjusting the remaining control such the thermal comfort is provided and that the external disturbance rejected. The objective of this paper is to

- design a control that ensures desired performance,

- design a reconfigurable control using the remaining input control that compensates for a stuck damper fault and eliminates the external disturbance effect for the faulty system.

In the next section, the synergetic control strategy is presented for HVAC system control.

\section{Synergetic control for HVAC system control}

3.1. Brief review of the synergetic approach. Consider the nonlinear dynamical system

$$
\dot{x}=A(x)+B(x) u,
$$

where $x \in \mathbb{R}^{n}$ is the state variable, $u \in \mathbb{R}^{m}$ is the control input and $A(x)$ and $B(x)$ are matrices with appropriate dimensions. The main principle of synergetic control is to steer the state trajectories towards a specific trajectory called the manifold. It is a two-step approach, starting with the selection of the manifold which can be a function of a state vector as follows:

$$
s(x)=x-x_{\text {ref }}, \quad s(x) \in \mathbb{R}^{m},
$$

$s(x)$ being designed so that $\frac{\partial s(x)}{\partial x} B(x)$ is invertible.

The manifold is designed according to performances and control specifications. The synergetic control objective is to force the system to reach the manifold, and the dynamics evolution of the manifold $s(x)=0$ are imposed as

$$
T \dot{s}(x)+s(x)=0,
$$

where $T=T^{T}>0$ is the designed speed convergence parameter towards the desired manifold.

The derivative of the manifold is given by

$$
\dot{s}(x)=\frac{\partial s(x)}{\partial x} \cdot \frac{\partial}{\partial t}\left(x-x_{\mathrm{ref}}\right)=\dot{x} .
$$

If $x_{\text {ref }}$ is constant, then $\dot{x}_{\text {ref }}=0$. The synergetic control law is obtained by solving (11) as follows:

$$
\begin{aligned}
T \dot{s}(x)+s(x) & =T \dot{x}+s(x) \\
& =T\left(A(x)+B(x) u_{\mathrm{syn}}\right)+s(x)=0 .
\end{aligned}
$$

Thus,

$$
\begin{aligned}
u_{\mathrm{syn}} & =-(T B(x))^{-1}(T A(x)+s(x)) \\
& =-(T B(x))^{-1} A(x)-(T B(x))^{-1}(s(x)) \\
& =u_{\mathrm{eq}}+u_{c}
\end{aligned}
$$

with

$$
\begin{gathered}
u_{\mathrm{eq}}=-(T B(x))^{-1} T A(x), \\
u_{c}=-(T B(x))^{-1} s(x) .
\end{gathered}
$$

When $T=1$, the synergetic control law is similar to the form of the sliding mode control law that is obtained by solving $\dot{s}(x)=0$ for $u$ :

$$
\dot{s}(x)=\dot{x}=A(x)+B(x) u_{\mathrm{eq}}=0 .
$$

Then

$$
u_{\mathrm{eq}}=-(B(x))^{-1} A(x) .
$$

The variable-structure sliding-mode control law has the following form (Slotine and $\mathrm{Li}, 1991$ ):

$$
u_{\mathrm{SMC}}=u_{\mathrm{eq}}+u_{d}
$$

with $u_{d}=-k(B(x))^{-1} \operatorname{sign}(s(x))$, where $k$ is the controller gain and it is a freedom design parameter. A simple comparison between (14) and (16) proves that both controllers have the same equivalent control law $u_{\text {eq }}$ that drives the state trajectories towards the manifold; $u_{d}$ and $u_{c}$ are used to maintain the system dynamics along the manifold $s(x)=0$. However, the sliding mode control law comprises a discontinuous term $u_{d}$, which induces the chattering phenomenon.

The advantage of the synergetic control law over the sliding-mode controller is that it includes a continuous term which provides stable control without oscillations. It can be observed also that $u_{c}$ is a linear continuous approximation to $u_{d}$. Thereafter, synergetic control can be a solution to overcome the chattering phenomenon of sliding mode control. In this paper, the design of synergetic control uses the nonlinear model of the HVAC system. The imposed constraint (11) reduces the system order, ensuring global stability and robustness to disturbance and parameter variation. Briefly, an adequate choice of the manifold ensures the performance sought and global stability. The stability of a synergetic controller is proved by the following theorem. 
Theorem 1. Consider the nonlinear system (9) and a positive definite diagonal matrix $T$. The system states driven by the control law (14) will converge exponentially to the origin with the rate of convergence depending on the selected parameter $T$.

Proof. Define the Lyapunov function as follows:

$$
V(s(x))=s(x)^{T} s(x) .
$$

Its derivative is given as

$$
\frac{\mathrm{d}}{\mathrm{d} t} s(x)^{T} s(x)=2 s(x)^{T} \dot{s}(x) .
$$

From (11) we have $\dot{s}(x)=-T^{-1} s(x)$. Thus

$$
\begin{aligned}
\frac{\mathrm{d}}{\mathrm{d} t} s(x)^{T} s(x) & =-2 s(x)^{T} T^{-1} s(x) \\
& =-2 T^{-1}\|s(x)\|<0 \text { for } s(x) \neq 0 .
\end{aligned}
$$

Hence, based on the Lyapunov approach, the controlled system (9) with the control input (14) is stable.

In what follows, we will prove the robustness of the synergetic controller to disturbance.

3.2. Robustness of synergetic control against external disturbance. The nonlinear dynamic model with external disturbance is described by

$$
\dot{x}=A(x)+B(x) u+E d(t),
$$

where $d(t)$ is the time-varying external disturbance and $E \in \mathbb{R}^{n}$ is the disturbance distribution matrix.

Theorem 2. Consider the nonlinear system (21) and time-varying external disturbance $d(t)$ that satisfies the matched condition. The synergetic control law can reject the external disturbance.

Proof. Applying the synergetic approach to the above nonlinear system, we obtain the following control law:

$$
u_{\mathrm{syn}}=-(T B(x))^{-1}(T A(x)+T E d(t)+s(x)) .
$$

It is shown that the resulting control law depends on the disturbance. Thus, the system dynamics restricted to the manifold $s(x)=0$ are given as follows:

$$
\begin{aligned}
\dot{x}= & A(x)+B(x) u_{\text {syn }}+E d(t) \\
= & A(x)-B(x)\left((T B(x))^{-1}(T A(x)+T E d(t)\right. \\
& +s(x)))+E d(t) .
\end{aligned}
$$

Suppose that the external disturbance can be defined as $D=E d(t)$ and it satisfies the matching condition. A disturbance satisfies the matching condition if it is within the range of input function $B(x)$ and upper bounded (Utkin, 2013; Jafarov, 2005; Veselić et al., 2014). Thus, a matched disturbance must satisfy the following conventional matching conditions. There exists function $\beta(x, t)$ such that the external disturbance that satisfies the matching condition can be written as

$$
E d(t)=B(x) \beta(x, d)
$$

Then, the system dynamics are expressed as

$$
\begin{gathered}
\dot{x}=A(x)-B(x)\left(\begin{array}{c}
(T B(x))^{-1}(T A(x) \\
+T B(x) \beta(x, d)+s(x))
\end{array}\right) \\
+B(x) \beta(x, d), \\
\dot{x}=\left(I_{n}-B(x)(T B(x))^{-1}\right) T A(x) \\
-B(x)(T B(x))^{-1} s(x) .
\end{gathered}
$$

From Eqn. 26 it is clear that the system dynamics on the manifold are independent of the external disturbance, which proves the robustness of synergetic control to the disturbance. These analytical results will be verified in the simulation section.

Remark 1. Note that if we have a system affected by an unknown and unmatched disturbance, it is proved that the synergetic controller is robust against this disturbance according to the analytical design of aggregated regulators (ADAR) method of Kuz'menko et al. (2015).

In the next section, we will illustrate the above discussion with the HVAC system.

3.3. Design of synergetic control for the HVAC system. In this section, the above synergetic theories are used to ensure desired HVAC system performances. As mentioned above, the desired performance of a multi-zone building system consists in providing a comfortable temperature in the zone through the air flow rates. The manipulated variables for this system are the controls $u_{1}, \ldots, u_{N}$. Our objective now is to design synergetic controllers that force the zone temperatures $x_{1}, \ldots, x_{N}$ to converge respectively towards the desired signals $x_{\text {ref }_{1}}, \ldots, x_{\operatorname{ref}_{N}}$. To this end, we propose the following manifold:

$$
s(x)=\left[\begin{array}{lll}
s_{1}(x) & \ldots & s_{N}(x)
\end{array}\right]^{T},
$$

with

$$
s_{i}(x)=x_{i}-x_{\operatorname{ref}_{i}}, \quad 1 \leq i \leq N .
$$


Remark 2. The reference temperatures $x_{\mathrm{ref}_{1}}, \ldots, x_{\mathrm{ref}_{N}}$ depend on the occupancy of the zone: when the zone is empty, we choose reference values as $x_{r e f_{i}}=18^{\circ} \mathrm{C}$, while if totally occupied, the required value is $x_{\text {ref }_{i}}=$ $22^{\circ} \mathrm{C}$. Therefore, the reference temperatures are a signal range between $18^{\circ} \mathrm{C}$ and $22^{\circ} \mathrm{C}$ according to the occupancy gain $g$. Thus, the reference value $x_{\text {ref }}$ is piecewise constant.

Solving the first-order differential equation (11) for $u$ yields a controller that drives the state variable to lie on the manifold $s(x)=0$. To proceed, let

$$
T \dot{s}(x)+s(x)=0,
$$

where $T>0$ is a symmetric definite positive matrix having the form $T=\operatorname{diag}\left[\begin{array}{lll}T_{11} & \ldots & T_{N N}\end{array}\right]$. Note that for the design of speed convergence parameters $T$, it has to take into account the feasibility of control. We derive nonlinear control laws $u_{\text {syn }}=\left[u_{1 \text { syn }}, \ldots, u_{\text {Nsyn }}\right]^{T}$ that force the system to operate in a neighbourhood of manifolds $s_{1}(x)=0, \ldots, s_{N}(x)=0$ from the above equations. The synergetic control law for the zone is obtained by differentiating (30) while taking into account (7) as follows:

$$
\begin{aligned}
T \dot{s}(x)+s(x)= & T \dot{x}+s(x) \\
= & T\left(A_{N}(x)+B_{N}(x) u_{\text {syn }}\right. \\
& \left.+E_{N} d(t)\right)+s(x)=0,
\end{aligned}
$$

where the matrices $A_{N}(x), \quad B_{N}(x)$ and $E_{N}$ are matrices associated with the state variable $\left[\begin{array}{llll}x_{1} & x_{2} & \ldots & x_{N}\end{array}\right]^{T}$. Then,

$$
\begin{aligned}
u_{\text {syn }}= & -\left(T B_{N}(x)\right)^{-1}\left(T A_{N}(x)+T E_{N} d(t)\right. \\
& +s(x))
\end{aligned}
$$

For the HVAC system, synergetic control is feasible if $T B_{N}(x)$ is invertible.

In the nominal case, the air temperature controller $u_{N+1}$ can be considered constant. It is shown in the above equation that synergetic control laws depend on the designed parameter $T$, the manifolds and the external disturbance. The control laws $u_{1 \text { syn }}, \ldots, u_{\text {Nsyn }}$ drive the system trajectory to satisfy Eqn. (11). According to this equation, the state-variable trajectories converge to the manifolds $s_{1}(x)=0, \ldots, s_{N}(x)=0$, respectively with the design convergence parameters $T_{11}, \ldots, T_{N N}$. Then they remain along the manifolds at all future times. Substituting the control law (31) into the system differential equations (7), we obtain the systems dynamics along the manifolds as follows:

$$
\begin{aligned}
\dot{x}= & A_{N}(x)+B_{N}(x) u_{\text {syn }}+E_{N} d(t) \\
= & A_{N}(x)-B_{N}(x)\left(T B_{N}(x)\right)^{-1}\left(T A_{N}(x)\right. \\
& \left.+T E_{N} d(t)+s(x)\right)+E_{N} d(t)
\end{aligned}
$$

If $B_{N}(x)$ is invertible, then

$$
\dot{x}=-\frac{s(x)}{T} .
$$

A suitable value of convergence parameter $T$ provides an optimal synergetic control that minimizes energy consumption.

All these theoretical results will be verified in the simulation section. Now we will study the behaviour of synergetic control when a fault occurs. We will show its intervention to compensate for the fault.

\section{Synergetic control for compensating for a stuck damper fault}

In this section, we show that synergetic control can compensate for a fault and recover the system to the nominal operation. When actuators are stuck, the corresponding control signal will be constant. Thus, the nominal controller will no longer perform the task of maintaining the performance sought. Consider the system (7) and let the damper of the zone get stuck. Thus, control input $u_{i}$ becomes constant. The effect of a stuck damper can be presented as an additional constant disturbance imposed onto the nominal system for the stuck damper, system (6) can be written as

$$
\dot{x}=A(x)+B_{0}(x) u_{0}+B_{f}(x) u_{f}+E d(t),
$$

with $B_{f}(x)=b_{1}(x)$, where $B_{0}(x)$ is the column associated to the healthy controllers, $u_{f}$ is a constant that gives the value at which the damper is stuck and $u_{0}$ denotes the remaining control input.

For the faulty system the matched condition is not fulfilled because of the null row of matrix $B_{f}(x)$. In fact, the null row is due to a stuck damper fault which makes the associated control constant, and thereafter it will be considered an additive disturbance and not a control input. It is assumed that the fault value is determined using one of the existing FDD techniques presented by Kim and Katipamula (2018), and thus it is available for designing a reconfigurable control. We also use a commutation mechanism or a decision mechanism after the block of the fault estimation to ensure activation of the reconfigurable controller. The mechanism of commutation can be defined as follows.

Let the thresholds $f_{\text {th }}$ that is chosen indicate the activation of the reconfigurable controller as follows:

$$
u_{4}= \begin{cases}u_{4 \mathrm{nom}}, & \hat{u}_{f}<f_{\mathrm{th}}, \\ u_{r}, & \hat{u}_{f} \geq f_{\mathrm{th}},\end{cases}
$$

where $\hat{u}_{f}$ can be considered fault estimate obtained through the block of FDD. The procedure of choosing the thresholds is complicated and many methods have 
been developed to select the appropriate thresholds; for more details, see Patton et al. (1989). Here we design a reconfigurable controller by adjusting the air temperature controller $u_{N+1}$ using the synergetic approach in order to provide a suitable air temperature $x_{\text {ref }_{N+1}}$ so that the temperature in the zones remains constant despite the blocking of the dampers.

Synergetic theory is mainly based on the manifold and the first-order differential equation (11). Indeed, a suitable choice of the manifold by the designer drives the system to the desired path. Thus, the desired system performances are imposed through the new manifold $s_{N+1}(x)$, which is designed to construct a reconfigurable synergetic control $u_{r}$. Thus the initial performance can be recovered using the control $u_{r}$ that is synthesized using synergetic control so that the air temperature $x_{N+1}$ converges to the reference value $x_{\mathrm{ref}_{N+1}}$. Here $x_{\mathrm{ref}_{N+1}}$ is obtained so that the temperature in Zone $i$ in the faulty case is equal to the reference signal.

Now we suppose that the temperature of Zone $i$ is driven by the nominal synergetic controller, and its dynamics along the manifold $s_{i}(x)$ are given by Eqns. (36) and (37). Our objective is to determine the expression of $x_{\text {ref }_{N+1}}$ that will maintain the temperature of Zone 1 in the reference signal. That is why we assume that the state variable $x_{i}$ is in the steady state. In steady state the state variable $x_{i}$ is equal to the reference signal. Hence, according to this assumption, the temperature of Zone $i$ and its dynamics along the manifold $s_{i}(x)$ are given by the following equations:

$$
\begin{gathered}
x_{i}=x_{\text {ref }_{i}}, \\
\dot{x}_{i}=-\frac{s_{i}(x)}{T_{i}}=0 .
\end{gathered}
$$

Then, our objective is to design a feedback controller $u_{r}$ that maintains the temperature of Zone $i$ in the initial performance. The control $u_{r}$ is obtained by adjusting the air temperature controller $u_{N+1}$. To this end, let us introduce a new manifold,

$$
s_{N+1}(x)=x_{N+1}-x_{\text {ref }_{N+1}},
$$

where $x_{\mathrm{ref}_{N+1}}$ is the reference value of the air temperature $x_{N+1}$, and its expression is extracted from the above conditions (36) and 37) when a stuck damper fault occurs. It has the following form:

$$
x_{\mathrm{ref}_{N+1}}=f\left(x_{\mathrm{ref}_{i}}, d(t), x_{\mathrm{ref}_{i-1}}, x_{\mathrm{ref}_{i+1}}, u_{f}\right),
$$

where $x_{\text {ref }_{N+1}}$ forces the temperature of the affected Zone $i$, which is affected, to stay along the reference value. The control $u_{r}$ drives the air temperature $x_{N+1}$ to converge towards the reference $x_{\text {ref }_{N+1}}$. It is designed by solving the synergetic differential equation

$$
T_{N+1} \dot{s}_{N+1}(x)+s_{N+1}(x)=0 .
$$

We derive the manifold using (5) and obtain

$$
\begin{aligned}
& T_{N+1} \dot{x}_{N+1}-T_{N+1} \dot{x}_{\mathrm{ref}_{N+1}}+s_{N+1}(x) \\
& =T_{N+1}\left(-\frac{1}{\tau} x_{N+1}+\frac{\alpha}{\tau} u_{r}\right)-T_{N+1} \dot{x}_{\mathrm{ref}_{N+1}} \\
& \quad+s_{N+1}(x)=0 .
\end{aligned}
$$

Solving this equation for $u_{r}$, the reconfigurable synergetic control is

$$
u_{r}=-\frac{\tau}{T_{N+1} \alpha}\left[\begin{array}{l}
-\frac{T_{N+1}}{\tau} x_{N+1} \\
-T_{N+1}^{2} \dot{x}_{\operatorname{ref}_{N+1}}+s_{N+1}(x)
\end{array}\right] .
$$

Thus the above reconfigurable control law is designed in a faulty case in order to compensate for a stuck damper fault and recover the nominal operation in Zone $i$. It drives the air temperature to obtain the reference value, which in turn maintains the temperature in the faulty zone in the desired performance.

Remark 3. The reconfigurable control is activated automatically once Damper $i$ is stuck. Then the flow air becomes constant, so reconfigurable control is activated if we have $u_{i}=$ const.

Note that reconfigurable control $u_{r}$ is used to compensate the fault that affects the damper of Zone $i$; for the other zones we use the same control as in the previous section, constructed through manifolds, $s_{1}(x), \ldots, s_{i-1}(x), s_{i+1}(x), \ldots, s_{N}(x)$.

The main results are underlined and illustrated through a simulation example in the following section.

\section{Simulation results}

In this section, we simulate the analytical results presented above for a three-zone building HVAC system. To set up the simulation, we used the Matlab/Simulink component library. The time scale of the simulation is in hours. The parameters of the HVAC system are defined in Tables 1. Each of the three zones had an equal floor area of $30 \mathrm{~m}$ $\times 10 \mathrm{~m}$ and the wall was $3 \mathrm{~m}$ tall. Each zone had 10 windows, each $2 \mathrm{~m}$ wide and $2 \mathrm{~m}$ tall. All simulations were performed during 5 days in winter.

The damper of the VAV box of Zone 1 was stuck at the beginning of the second day. Occupation in the zones was different. Zone 1 was totally occupied during all days. Zone 2 was occupied only the first and the last day. Zone 3 was occupied only in the mornings. The time step size was selected as 1 hour, so that the total simulation time was $120 \mathrm{~h}$. The initial values of temperature were fixed at $10^{\circ} \mathrm{C}$. To assimilate the settings to the practical case, the controls $u_{1}, u_{2}, u_{3}$ in the simulation are expressed by percentages of damper openings. Thus the control input illustrated in the following figures is given by $u_{i}=$ $C \dot{m}_{i}$ for $1<i<N$, where $C$ is a constant gain that 


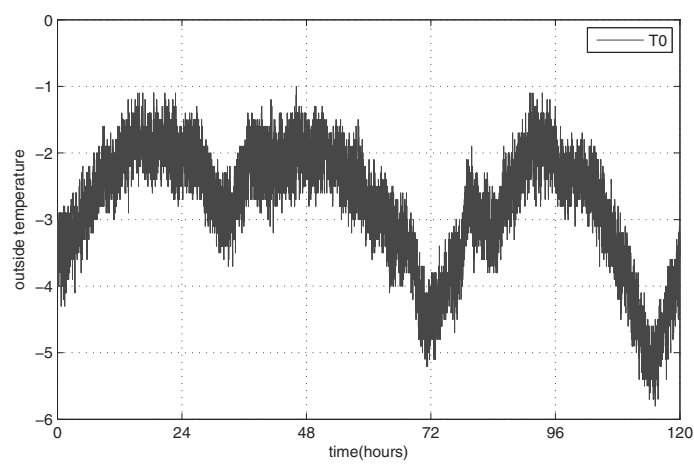

Fig. 2. Outside temperature.

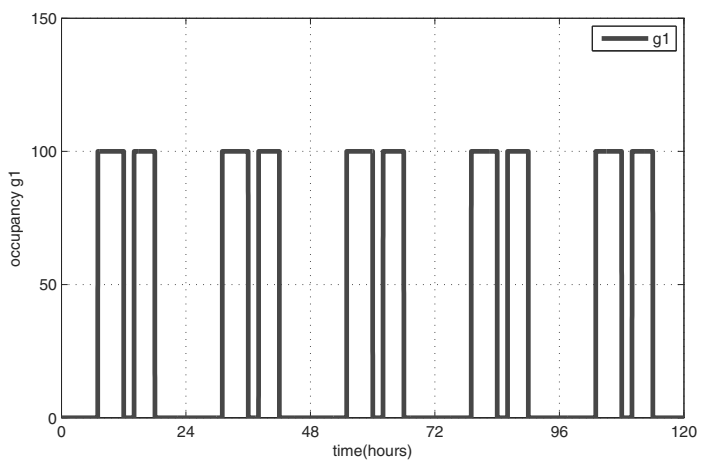

Fig. 3. Occupancy gain for Zone 1.

determines the percentage of the opening for the damper corresponding to the flow air.

Figure 2 shows the outside temperature variation during five days. Figures $3-5$ show the occupancy gain of each zone.

5.1. Synergetic control of a three-zone building. For the sake of simplicity, the synergetic controller is designed here for a building with three zones. The manipulated variables for this system are the controls $u_{1}, u_{2}$ and $u_{3}$. The synergetic controller was constructed by solving the differential equation $T \dot{s}(x)+s(x)=0$. In this work,

Table 1. HVAC system parameters.

\begin{tabular}{|c|c|}
\hline parameter & value \\
\hline \hline$M_{1}=M_{2}=M_{3}$ & $1470 \mathrm{~J} / \mathrm{K}$ \\
\hline$R_{1}=R_{2}=R_{3}$ & $1.8339 \mathrm{e}-07$ \\
\hline$R_{12}=R_{23}$ & $2.8935 \mathrm{e}-07$ \\
\hline$c_{p}$ & $1005.4 \mathrm{~J} / \mathrm{kg}-\mathrm{K}$ \\
\hline$\tau$ & 0.3 \\
\hline$\alpha$ & 0.7 \\
\hline
\end{tabular}

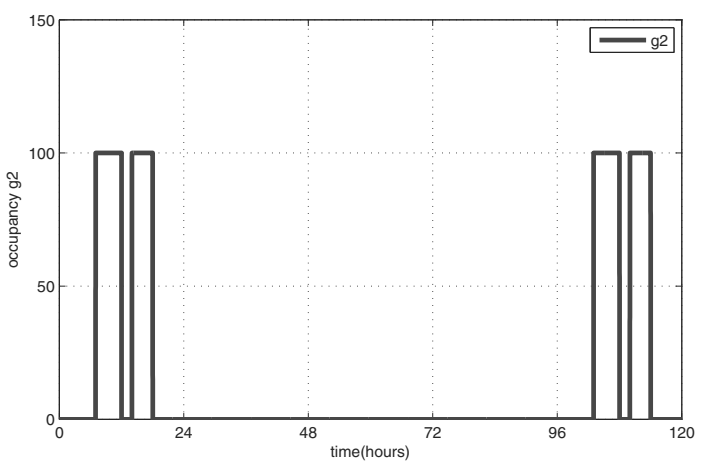

Fig. 4. Occupancy gain for Zone 2.

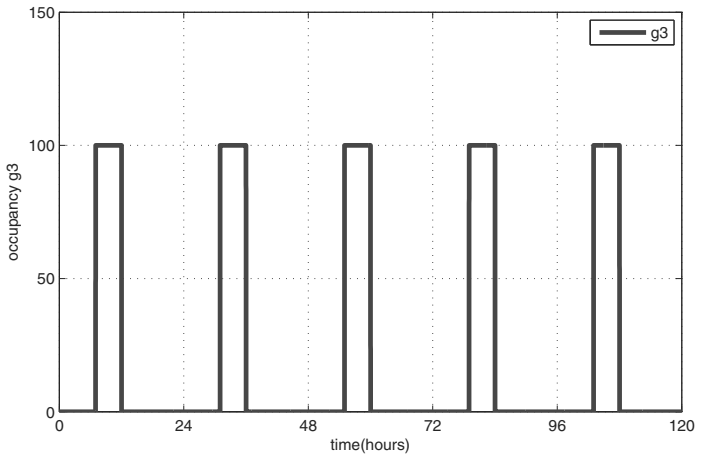

Fig. 5. Occupancy gain for Zone 3.

we designed three manifolds $s_{1}(x), s_{2}(x)$ and $s_{3}(x)$ and three convergence parameters $T_{11}, T_{22}$ and $T_{33}$. First, we selected $T_{11}=T_{22}=T_{33}=1$. The HVAC state space model given by (21) can be rewritten as follows:

$$
\begin{aligned}
\dot{x}_{1}= & \frac{u_{1}}{M_{1}}\left(x_{4}-x_{1}\right)-\frac{x_{1}}{M_{1} c_{p} R_{1}}+\frac{x_{2}-x_{1}}{M_{1} c_{p} R_{12}} \\
& +\frac{1}{M_{1} c_{p} R_{1}} T_{\text {out }}+\frac{1}{M_{1} c_{p}} g_{1}, \\
\dot{x}_{2}= & \frac{u_{2}}{M_{2}}\left(x_{4}-x_{2}\right)-\frac{x_{2}}{M_{2} c_{p} R_{2}}+\frac{x_{1}-x_{2}}{M_{2} c_{p} R_{12}} \\
& +\frac{x_{3}-x_{2}}{M_{2} c_{p} R_{23}}+\frac{1}{M_{2} c_{p} R_{2}} T_{\text {out }}+\frac{1}{M_{2} c_{p}} g_{2}, \\
\dot{x}_{3}= & \frac{u_{3}}{M_{3}}\left(x_{4}-x_{3}\right)-\frac{x_{3}}{M_{3} c_{p} R_{3}}+\frac{x_{2}-x_{3}}{M_{3} c_{p} R_{32}} \\
& +\frac{1}{M_{3} c_{p} R_{3}} T_{\text {out }}+\frac{1}{M_{3} c_{p}} g_{3} .
\end{aligned}
$$

The HVAC building system is affected by the disturbance $d(t)$. Therefore, after starting the development of synergetic control, we have to prove that 
the disturbance satisfies the condition

$$
E d(t)=B(x) \beta(x, d),
$$

where $E=\left[\begin{array}{ll}E_{1} & E_{2}\end{array}\right], d=\left[\begin{array}{ll}T_{\text {out }} & g\end{array}\right]^{T}$ and we have

$$
\begin{aligned}
& B(x)=\left(\begin{array}{cccc}
\frac{\left(x_{4}-x_{1}\right)}{M_{1}} & 0 & 0 & 0 \\
0 & \frac{\left(x_{4}-x_{2}\right)}{M_{2}} & 0 & 0 \\
0 & 0 & \frac{\left(x_{4}-x_{3}\right)}{M_{3}} & 0 \\
0 & 0 & 0 & \frac{\alpha}{\tau}
\end{array}\right), \\
& E_{1}=\left(\begin{array}{c}
\frac{1}{M_{1} c_{p} R_{1}} \\
\frac{1}{M_{2} c_{p} R_{2}} \\
\frac{1}{M_{3} c_{p} R_{3}} \\
0
\end{array}\right) \\
& E_{2}=\left(\begin{array}{ccc}
\frac{1}{M_{1} c_{p}} & 0 & 0 \\
0 & \frac{1}{M_{2} c_{p}} & 0 \\
0 & 0 & \frac{1}{M_{3} c_{p}} \\
0 & 0 & 0
\end{array}\right) \text {. }
\end{aligned}
$$

The matrices $E_{1}$ and $E_{2}$ can be rewritten as

$$
E_{1}=B(x) \Psi_{1}(x), \quad E_{2}=B(x) \Psi_{2}(x),
$$

where

$$
\begin{gathered}
\Psi_{1}(x)=\left(\begin{array}{c}
\frac{1}{c_{p} R_{1}\left(x_{4}-x_{1}\right)} \\
\frac{1}{c_{p} R_{2}\left(x_{4}-x_{2}\right)} \\
\frac{1}{c_{p} R_{3}\left(x_{4}-x_{3}\right)} \\
0
\end{array}\right), \\
\Psi_{2}(x)=\left(\begin{array}{ccc}
\frac{1}{c_{p}\left(x_{4}-x_{1}\right)} & 0 & 0 \\
0 & \frac{1}{c_{p}\left(x_{4}-x_{2}\right)} & 0 \\
0 & 0 & \frac{1}{c_{p}\left(x_{4}-x_{3}\right)} \\
0 & 0 & 0
\end{array}\right) .
\end{gathered}
$$

Hence we obtain

$$
\begin{aligned}
E d(t) & =E_{1} T_{\text {out }}+E_{2} G \\
& =B(x)\left(\Psi_{1}(x) T_{\text {out }}+\Psi_{2}(x) g\right) \\
& =B(x) \beta\left(x, T_{\text {out }}, g\right) .
\end{aligned}
$$

Our objective now is to design synergetic controllers that force the temperature of zones $x_{1}, x_{2}, x_{3}$ to converge respectively towards the desired signals $x_{\mathrm{ref}_{1}}, x_{\mathrm{ref}_{2}}, x_{\mathrm{ref}_{3}}$. To this end, we propose the following manifolds:

$$
s(x)=\left[\begin{array}{lll}
s_{1}(x) & s_{2}(x) & s_{3}(x)
\end{array}\right]^{T} .
$$

The synergetic controllers that drive the state variable to lie on the manifolds $s_{1}(x)=0, s_{2}(x)=0, s_{3}(x)=0$ are obtained by solving the equations

$$
\begin{aligned}
& T_{11} \dot{s}_{1}(x)+s_{1}(x)=0, \\
& T_{22} \dot{s}_{2}(x)+s_{2}(x)=0,
\end{aligned}
$$

$$
T_{33} \dot{s}_{3}(x)+s_{3}(x)=0,
$$

where $T_{11}>0, T_{22}>0, T_{33}>0$ are the designed convergence parameters associated with $s_{1}(x)=$ $0, s_{2}(x)=0, s_{3}(x)=0$, respectively. We take, for example, the synergetic control law for Zone 1 obtained by differentiating (48) taking into account (43) as follows:

$$
\begin{gathered}
T_{11}\left(\frac{u_{1 \mathrm{syn}}}{M_{1}}\left(x_{4}-x_{1}\right)-\frac{x_{1}}{M_{1} c_{p} R_{1}}+\frac{x_{2}-x_{1}}{M_{1} c_{p} R_{12}}\right. \\
\left.+\frac{1}{M_{1} c_{p} R_{1}} T_{\text {out }}+\frac{1}{M_{1} c_{p}} g_{1}\right)+s_{1}(x)=0,
\end{gathered}
$$

where $M_{i} / T_{i}\left(x_{4}-x_{i}\right)$ is invertible. Hence

$$
\begin{aligned}
u_{1 \text { syn }}= & -\frac{M_{1}}{T_{11}\left(x_{4}-x_{1}\right)}\left(-\frac{T_{11}}{M_{1} c_{p} R_{1}} x_{1}\right. \\
& +\frac{T_{11}}{M_{1} c_{p} R_{12}}\left(x_{2}-x_{1}\right)+\frac{T_{11}}{M_{1} c_{p} R_{1}} T_{\text {out }} \\
& \left.+\frac{T_{11}}{M_{1} c_{p}} g_{1}+s_{1}(x)\right) .
\end{aligned}
$$

The same approach holds for the other control laws:

$$
\begin{aligned}
u_{2 \text { syn }}= & -\frac{M_{2}}{T_{22}\left(x_{4}-x_{2}\right)}\left(-\frac{T_{22}}{M_{2} c_{p} R_{2}} x_{2}\right. \\
& +\frac{T_{22}}{M_{2} c_{p} R_{12}}\left(x_{1}-x_{2}\right)+\frac{T_{22}}{M_{2} c_{p} R_{23}}\left(x_{3}-x_{2}\right) \\
& \left.+\frac{T_{22}}{M_{2} c_{p} R_{2}} T_{\text {out }}+\frac{T_{22}}{M_{2} c_{p}} g_{2}+s_{2}(x)\right) \\
u_{3 \text { syn }}= & -\frac{M_{3}}{T_{33}\left(x_{4}-x_{3}\right)}\left(-\frac{T_{33}}{M_{3} c_{p} R_{3}} x_{3}\right. \\
& +\frac{T_{33}}{M_{3} c_{p} R_{23}}\left(x_{2}-x_{3}\right)+\frac{T_{33}}{M_{3} c_{p} R_{3}} T_{\text {out }} \\
& \left.+\frac{T_{33}}{M_{3} c_{p}} g_{3}+s_{3}(x)\right)
\end{aligned}
$$

Substituting the control laws in the system differential equations (43), we obtain the following systems dynamics along the manifolds:

$$
\begin{aligned}
\dot{x}_{1}= & \frac{u_{1 \mathrm{syn}}}{M_{1}}\left(x_{4}-x_{1}\right)-\frac{x_{1}}{M_{1} c_{p} R_{1}}+\frac{x_{2}-x_{1}}{M_{1} c_{p} R_{12}} \\
& +\frac{1}{M_{1} c_{p} R_{1}} T_{\text {out }}+\frac{1}{M_{1} c_{p}} g_{1} \\
= & -\frac{s_{1}(x)}{T_{11}}, \\
\dot{x}_{2}= & \frac{u_{2 \mathrm{syn}}}{M_{2}}\left(x_{4}-x_{2}\right)-\frac{x_{2}}{M_{2} c_{p} R_{2}}+\frac{x_{1}-x_{2}}{M_{2} c_{p} R_{12}} \\
& +\frac{x_{3}-x_{2}}{M_{2} c_{p} R_{23}}+\frac{1}{M_{2} c_{p} R_{2}} T_{\text {out }}+\frac{1}{M_{2} c_{p}} g_{2} \\
= & -\frac{s_{2}(x)}{T_{22}},
\end{aligned}
$$




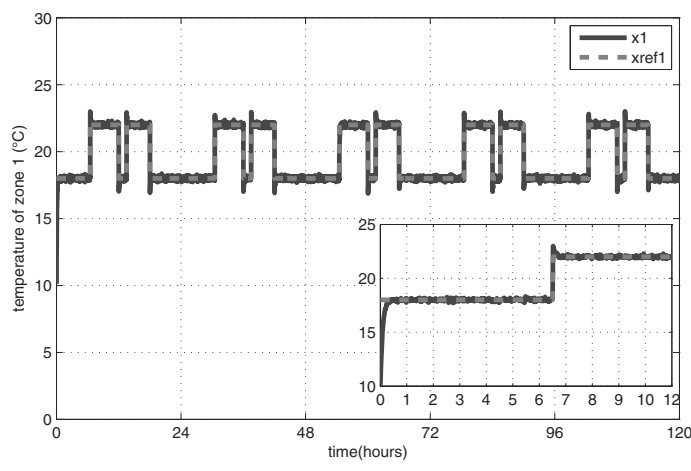

Fig. 6. Dynamics of the temperature of Zone 1 using synergetic control.

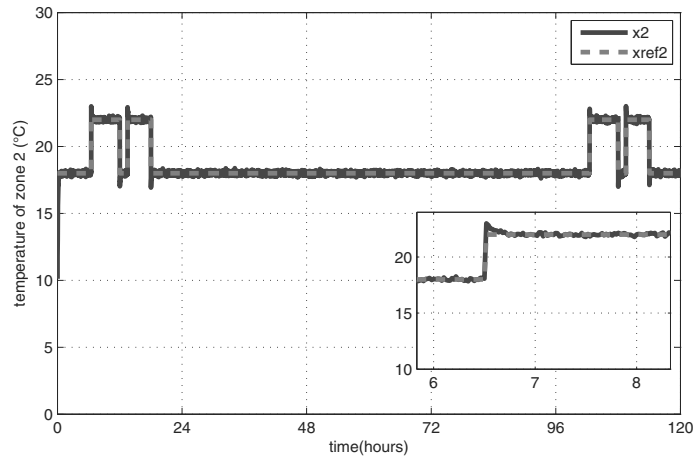

Fig. 7. Dynamics of the temperature of Zone 2 using synergetic control.

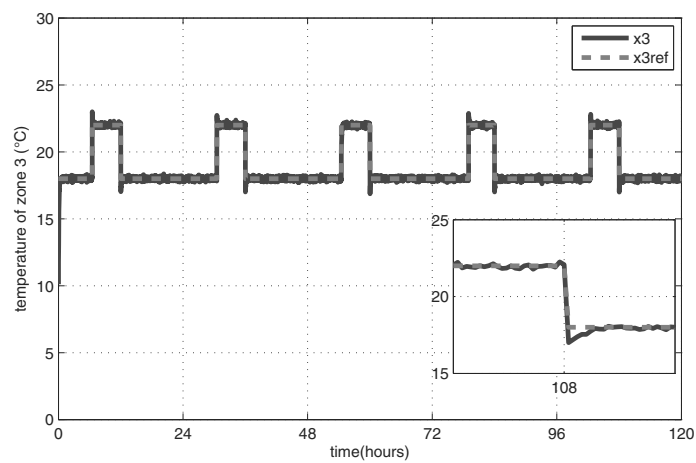

Fig. 8. Dynamics of the temperature of Zone 3 using synergetic control.

$$
\begin{aligned}
\dot{x}_{3}= & \frac{u_{3 \text { syn }}}{M_{3}}\left(x_{4}-x_{3}\right)-\frac{x_{3}}{M_{3} c_{p} R_{3}}+\frac{x_{2}-x_{3}}{M_{3} c_{p} R_{32}} \\
& +\frac{1}{M_{3} c_{p} R_{3}} T_{\text {out }}+\frac{1}{M_{3} c_{p}} g_{3} \\
= & -\frac{s_{3}(x)}{T_{33}} .
\end{aligned}
$$

Figures $6-8$ illustrate the closed-loop system response using synergetic control. They show the temperature response compared with the reference signals $x_{\text {ref }_{1}}, x_{\text {ref }_{2}}, x_{\text {ref }_{3}}$. Reference signals are plotted using dotted lines while the trajectories of temperature of the closed-loop HVAC system driven by the synergetic control laws (52), (53) and (54) are plotted using solid lines. As expected, we observe that the zone temperatures $x_{1}, x_{2}, x_{3}$ follow the imposed reference signal. Thus the closed-loop trajectories generated by synergetic control laws $u_{1 \text { syn }}, u_{2 \text { syn }}$ and $u_{3 \text { syn }}$ converge to the manifold $s_{1}(x), s_{2}(x)$ and $s_{3}(x)$. As can be seen from these figures, the synergetic closed-loop system responds rapidly to the change in the reference model, which is due to the variation in the occupancy in the zone. We also observe that the proposed control ensures total suppression of the disturbance effect (disturbance includes the external temperature and occupancy) to give a comfortable temperature inside each zone without oscillations induced by the disturbance. Therefore, the simulation results demonstrate the validity of the analytical findings in Section 3.

Figures $9-11$ show respectively the shapes of control signals $u_{1 \text { syn }}, u_{2 \text { syn }}$ and $u_{3 \text { syn }}$ given by (52), (53) and (54). For example, in Fig. 9 the control law almost takes the form of pulses with the disturbance distributed during 5 days that has the same form the distribution of the occupancy of Zone 1. This form demonstrates the dependence of the control law on disturbances. According to the temperature trajectories of each zone, they ensure compliance with the desired performances and suppression of disturbances. These controls allow the achievement of the desired goals. Each synergetic controller drives the corresponding temperature to reach the reference signal. Moreover, it rejects the effect of the disturbance, which is due to the outside temperature variation and the occupancy gain that yields a stable closed-loop system. Thus, we validate the results obtained in Section 3 to prove the robustness of synergetic control.

Now we will test the effect of the variation in the design parameter on the system dynamics. We take three values of $T: T=0.5, T=1$ and finally $T=10$. The following figures show the effect of the variation in the design parameter $T$ on the dynamics of the manifolds and system dynamics during 2 hours.

It is shown that, if the value of $T$ increases, the speed of convergence of the closed-loop dynamics driven by the synergetic controller toward the reference signal decreases. Thus the decreasing of the synergetic control law gain is proportional to the increasing of $T$.

To prove the effectiveness of our control compared to the existing methods, the synergetic controller (SC) is compared firstly with a linear LQR controller strategy that is widely used to control HVAC systems and then 


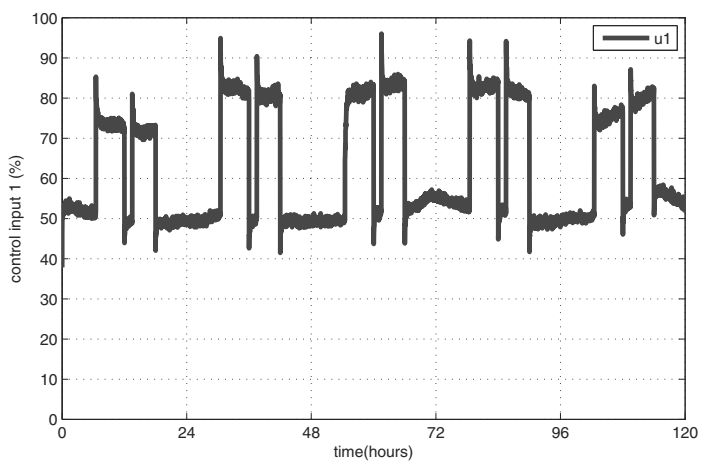

Fig. 9. Synergetic control for Zone 1.

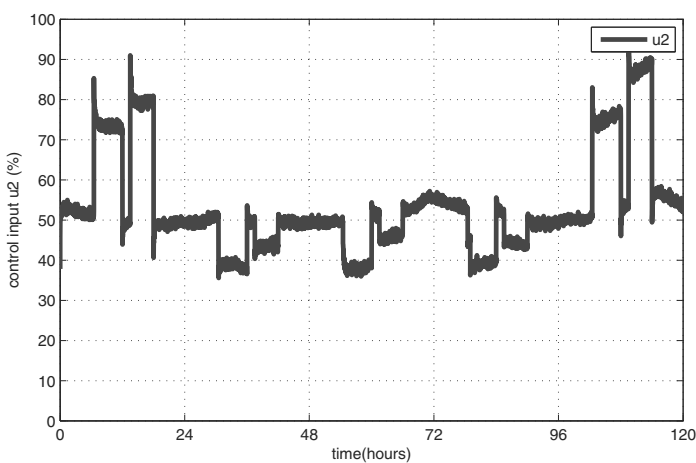

Fig. 10. Synergetic control for Zone 2 .

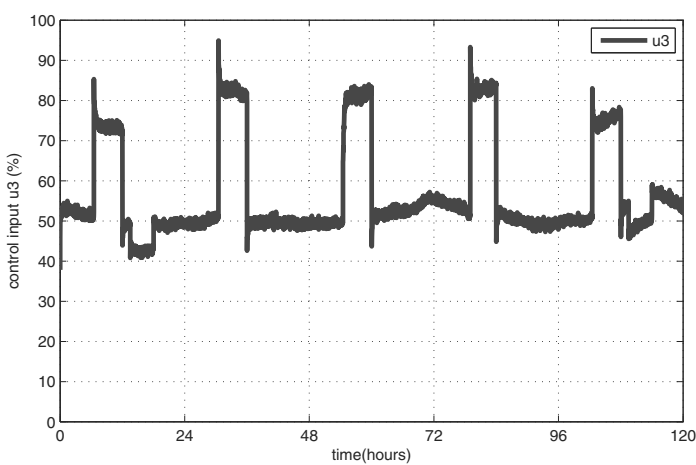

Fig. 11. Synergetic control for Zone 3.

with the sliding mode approach (SMC). We start by the comparison with LQR control; in Figs. 14 and 15 the solid line is the signal with the synergetic approach and the dotted line is the signal with the LQR approach. Figure 14 shows the dynamics of the temperature using the synergetic and the LQR controller. Figure 15 shows the controller input. From these figures, it is clear that both controllers force the zone temperature to converge

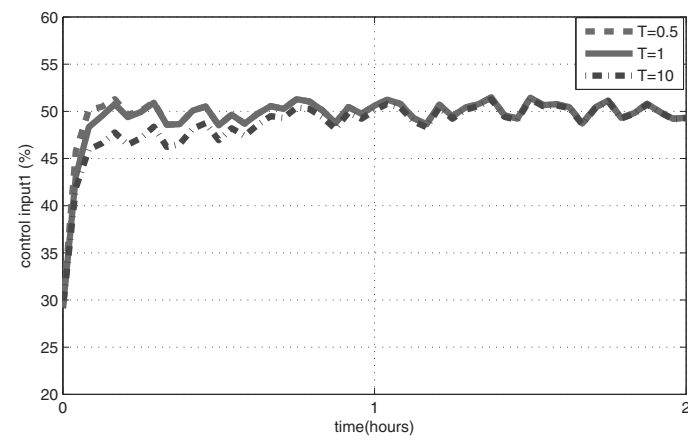

Fig. 12. Time evolution of the first control input.

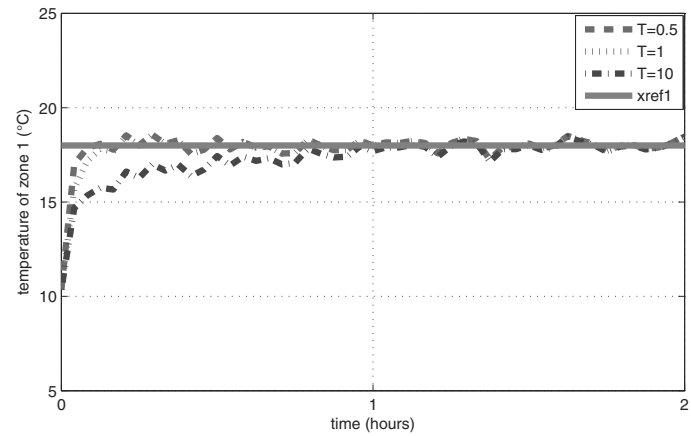

Fig. 13. Time evolution of temperature in Zone 1.

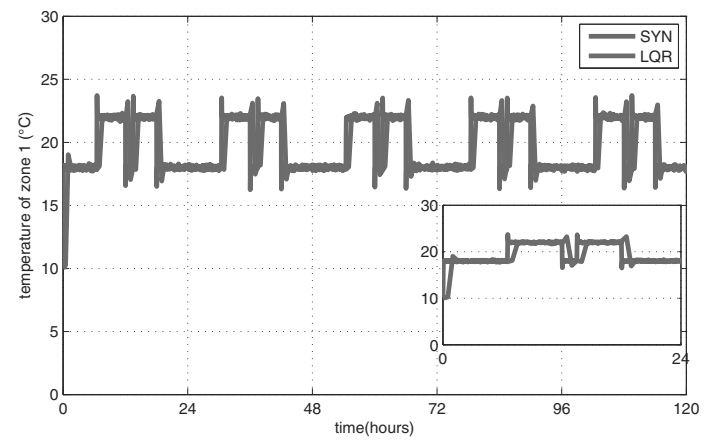

Fig. 14. Time evolution of temperature in Zone 1 using the linear and the synergetic controller.

to the reference signal. To compare the two approaches, we focus firstly on the response time of the synergetic controller compared with the LQR approach. It can be seen that the synergetic control signal converges rapidly to the reference signal compared with the LQR signal. We compared and investigated also the energy consumption. It is shown in Fig. 15 that sometimes (see, e.g., the first 24 hours) the energy consumption the synergetic 


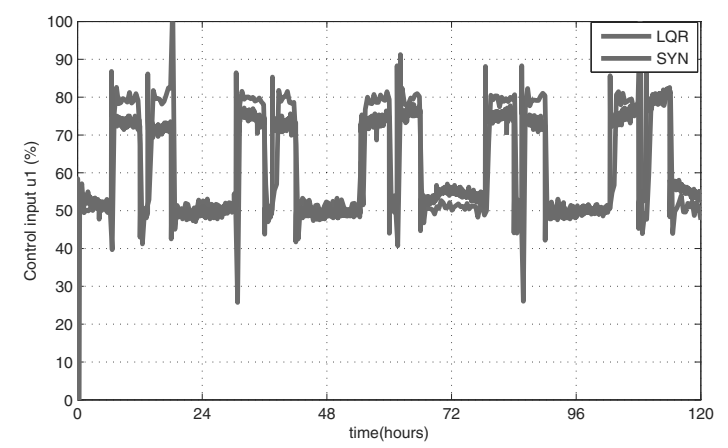

Fig. 15. Dynamics of both controllers.

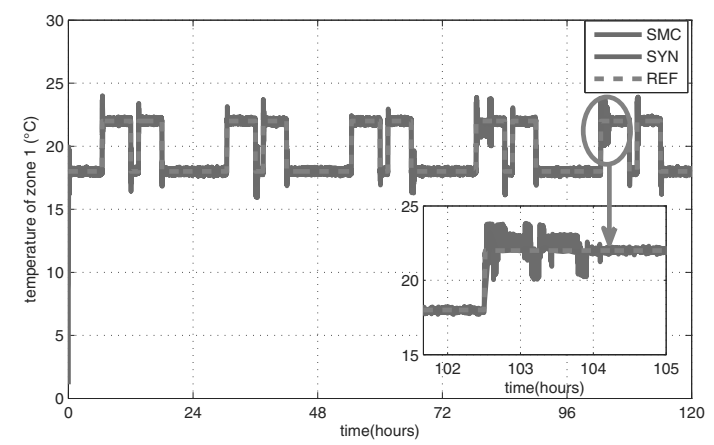

Fig. 16. Evolution of temperature in Zone 1 using the linear and the synergetic controller.

controller is smaller than that of the LQR controller. After the comparison between SC and LQR, we can conclude that synergetic control is more effective and more energy-efficient.

In order to prove the analytical results presented in the previous section, we compare the SC and SMC, which share the same principle to force the system trajectories to converge toward the manifold. In the next figures the dotted line is the signal with SC and the solid line shows the signal used with SMC.

Figure 16 shows the evolution of the zone temperature using both the synergetic approach and sliding mode control. In this figure, the dotted line is the signal with the synergetic approach, the solid line is the sliding mode control signal and the dash-dotted line is the reference signal. It can be seen that both controllers provide nearly the same performance. However, chattering is clearly visible in Figs. 16 and 17 while the signal given by the synergetic approach is free from chattering. In terms of robustness, the accuracy and disturbance rejection are assured by both the controllers. Now, if we compare the energy consumption for both the approaches, it is very similar except when the chattering

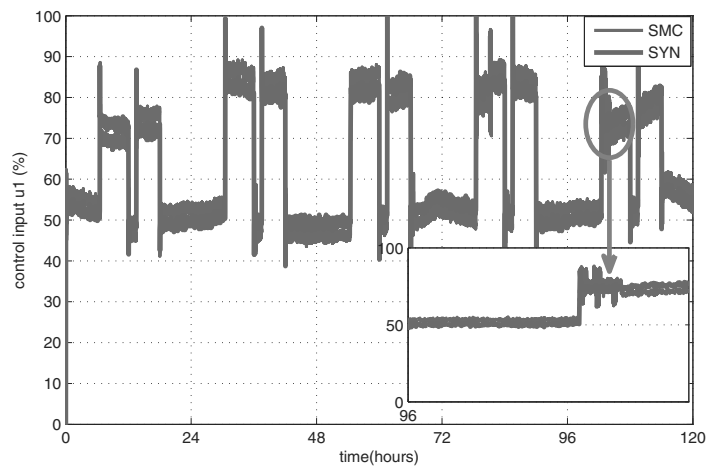

Fig. 17. Dynamics of both controllers.

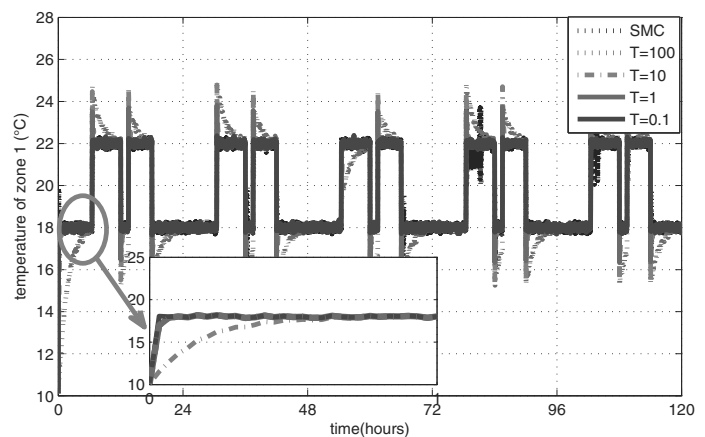

Fig. 18. Effect of the variation in $T$ on the dynamics of the temperature of Zone 1.

phenomenon appeared. Figure 18 shows the effect of variation in the value of $T$ on the zone temperature evolution. From this figure we can see that, when $T=1$, the dynamics of the temperature driven by the synergetic control resemble those driving by sliding mode control. This is because, for $T=1$, we have a term that yields a better approximation in the neighborhood of the manifold. Thereafter, the analytical results shown in the paper for linear approximation of sliding mode control are proved in the simulation. To conclude, synergetic control and sliding mode control have the same performances in terms of response time, robustness to the external disturbance and energy consumption, but synergetic control has the advantage of chattering elimination over sliding mode control.

5.2. Synergetic control for fault compensation. In this subsection, we suppose that the damper is stuck in the VAV box of Zone 1 at the beginning of the second day. Under the stuck damper the control $u_{1}$ becomes constant, $u_{1}=u_{f}=65 \%$. Thus it is no longer effective and the temperature in Zone 1 deviates from the reference model. The supply air flow has 
to be modified so that the temperature in Zone 1 is effectively restored in the desired performance, whereas the supply air temperature in the remaining zones is automatically adjusted correspondingly to this modified supply air temperature without affecting the temperature in the respective zones.

Consider the system (7); the damper of Zone 1 gets stuck. Thus control input $u_{1}$ becomes constant. Under the stuck damper, the matrices defined in the system (34) are given by

$$
\begin{gathered}
B_{f}(x)=\left(\begin{array}{c}
\frac{x_{4}-x_{1}}{M_{1}} \\
0 \\
0 \\
0
\end{array}\right), \\
B_{0}(x)=\left(\begin{array}{ccc}
0 & 0 & 0 \\
\frac{x_{4}-x_{2}}{M} & 0 & 0 \\
0 & \frac{x_{4}-x_{3}}{M} & 0 \\
0 & 0 & \frac{\alpha}{\tau}
\end{array}\right), \\
u_{0}=\left[\begin{array}{lll}
u_{2} & u_{3} & u_{4}
\end{array}\right]^{T} .
\end{gathered}
$$

First, let the manifold be $s_{4}(x)=x_{4}-x_{\mathrm{ref}_{4}}$. The reference value $x_{\text {ref }_{4}}$ is obtained using (36), (37) and (43):

$$
\begin{aligned}
\dot{x}_{1}= & \frac{u_{f}}{M_{1}}\left(x_{\mathrm{ref}_{4}}-x_{\mathrm{ref}_{1}}\right)-\frac{x_{\mathrm{ref}_{1}}}{M_{1} c_{p} R_{1}} \\
& +\frac{x_{\mathrm{ref}_{2}}-x_{\mathrm{ref}_{1}}}{M_{1} c_{p} R_{12}} \\
& +\frac{1}{M_{1} c_{p} R_{1}} T_{\text {out }}+\frac{1}{M_{1} c_{p}} g_{1}=0 .
\end{aligned}
$$

Solving the above for $x_{\mathrm{ref}_{4}}$ gives

$$
\begin{aligned}
x_{\mathrm{ref}_{4}}= & -\frac{M_{1}}{u_{f}}\left(-\frac{u_{f}}{M_{1}} x_{\mathrm{ref}_{1}}-\frac{x_{\mathrm{ref}_{1}}}{M_{1} c_{p} R_{1}}\right. \\
& +\frac{x_{\mathrm{ref}_{2}}-x_{\mathrm{ref}_{1}}}{M_{1} c_{p} R_{12}} \\
& \left.+\frac{1}{M_{1} c_{p} R_{1}} T_{\text {out }}+\frac{1}{M_{1} c_{p}} g_{1}\right)
\end{aligned}
$$

The first-order differential equation is $T_{44} \dot{s}_{4}(x)+s_{4}(x)=$ 0 . We derive the manifold using (5) and obtain

$$
T_{44}\left(-\frac{1}{\tau} x_{4}+\frac{\alpha}{\tau} u_{r}\right)-T_{44} \dot{x}_{\mathrm{ref}_{4}}+s_{4}(x)=0 .
$$

Solving this equation for $u_{r}$, reconfigurable synergetic control is

$$
u_{r}=-\frac{\tau}{T_{4} \alpha}\left[-\frac{T_{4}}{\tau} x_{4}-T_{44} \dot{x}_{\mathrm{ref}_{4}}+s_{4}(x)\right] .
$$

As mentioned above, reconfigurable control is activated if we have $\dot{u}_{i}=0$. Figures 19,21 and 23 illustrate the temperature responses after reconfiguration and the control laws are plotted in Figs. 20, 22 and 24.

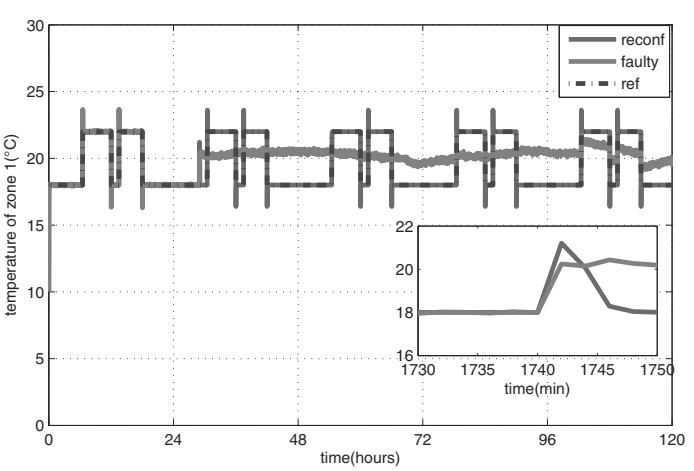

Fig. 19. Temperature of Zone 1 using reconfigurable control with a stuck damper fault.

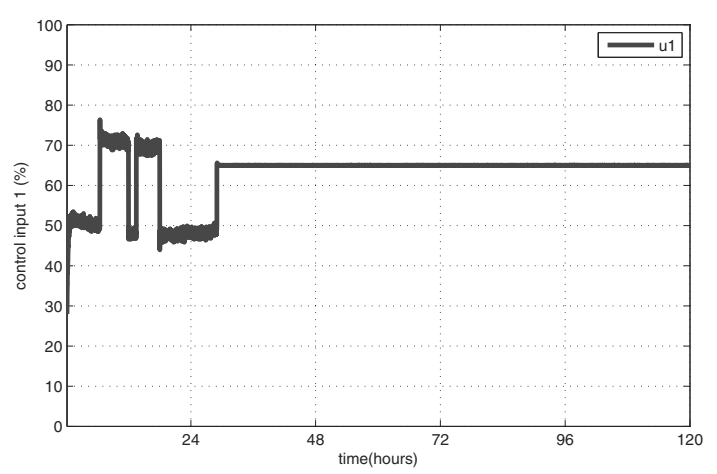

Fig. 20. Control $u_{1}$ for Zone 1 with a stuck damper fault in Zone 1.

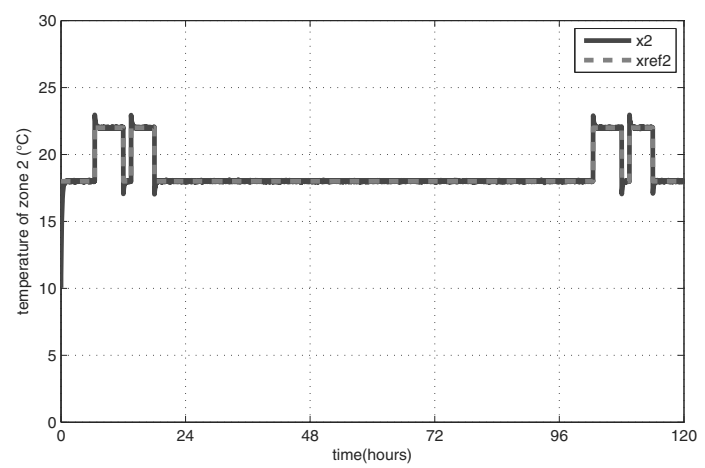

Fig. 21. Temperature of Zone 2 with a stuck damper fault in Zone 1.

As expected, the reconfigurable control $u_{r}$ activates automatically when a fault occurs. Synergetic reconfigurable control successfully compensates for damper stuck failure and recovers the original performance (Fig. 19). As can be seen from this figure, 


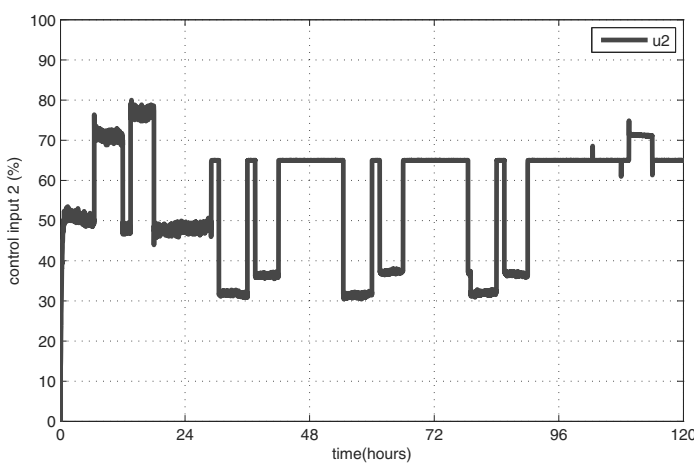

Fig. 22. Control $u_{2}$ with a stuck damper fault in Zone 1 .

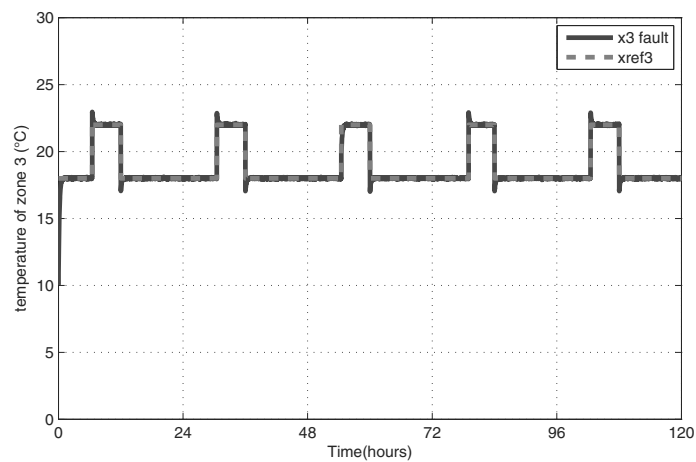

Fig. 23. Temperature of Zone 3 with a stuck damper fault in Zone 1.

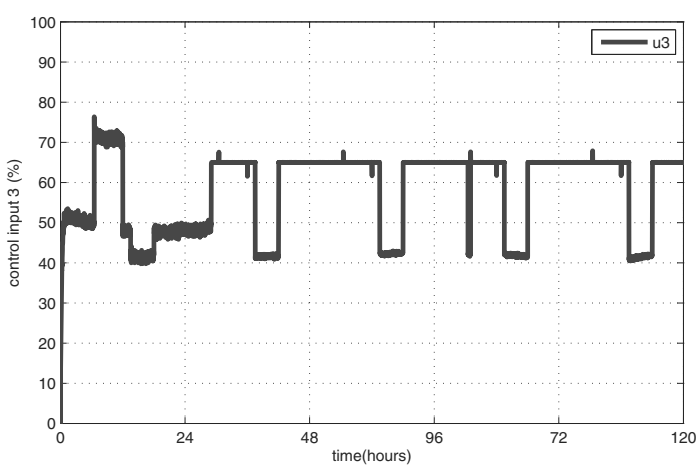

Fig. 24. Control $u_{3}$ with a stuck damper fault in Zone 1 .

reconfigurable control restores the temperature from a faulty value of $20^{\circ} \mathrm{C}$ to $18^{\circ} \mathrm{C}$. The temperature in Zone 1 is returned to the desired trajectory in 5 minutes. This reconfigurable control does not only compensate for the fault effect but also suppresses the unmatched disturbance effect. It generates a supply air temperature that leads to

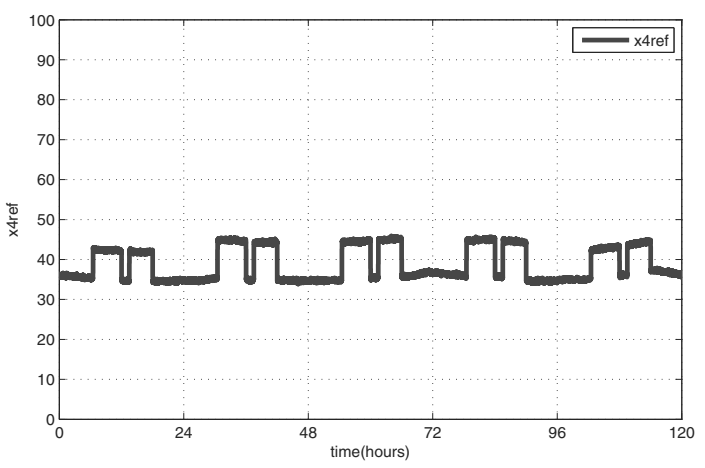

Fig. 25. Reference signal of the air temperature.

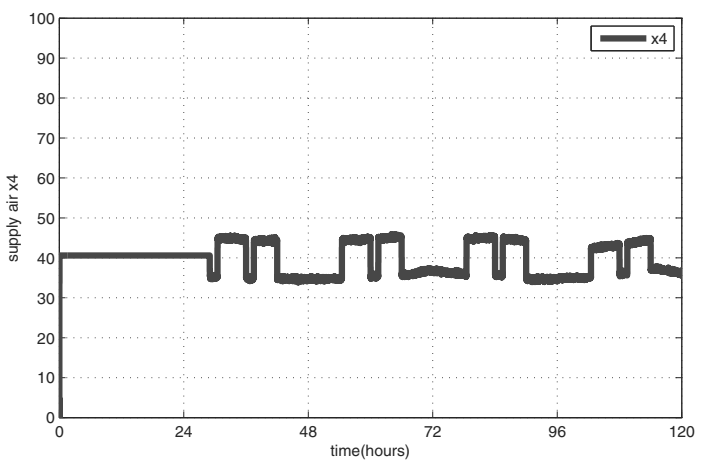

Fig. 26. Dynamics of the air temperature using reconfigurable control.

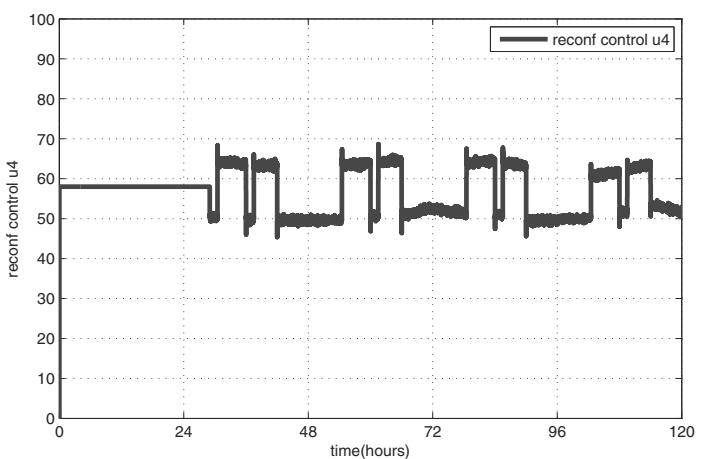

Fig. 27. Reconfigurable control.

disturbance suppression (Fig. 26). The stuck damper in Zone 1 does not affect the thermal comfort in Zones 2 and 3 (Figs. 21 and 23). However, the shape of control inputs $u_{2}$ and $u_{3}$ change when the fault occurs. This variation is due to the new supply air temperature.

Comparing Figs. 10 and 22 after using 
reconfigurable control there is no disturbance. This proves that the new supply air temperature compensates for the outside temperature. Figure 25 shows the reference of the supply air temperature. Figures 26 and 27 show respectively the supply air temperature and synergetic reconfigurable control. Before the damper is stuck, the supply air temperature is selected as $40^{\circ} \mathrm{C}$. Once a fault occurs, it becomes a signal that depends on the outside temperature and the occupancy gain $g_{1}$ and, also on the fault value and the reference temperature of Zone 1 .

\section{Conclusion}

Most control techniques use the linearized state space model around an equilibrium point. However, in this paper, synergetic control uses directly the nonlinear model, which is beneficial for the building HVAC community, because the linearization of the building model can degrade the control performance. The main advantage of the proposed synergetic control is in the ease of design and its high-quality control performance. Only one parameter to tune $T$ to provide good results is required, taking account the trade-off between robustness and performance. For synergetic control, the rate of convergence to the reference is controlled by the convergence parameter $T$, where a low value of $T$ gives a rapid rate of convergence to the desired value. However, in some cases, the implementation of the control law based on the synergetic approach has to fulfill some assumptions. In terms of robustness, synergetic control is insensitive to a noise and ensures disturbance rejection. When a fault occurs, reconfigurable synergetic control was successful in recovering the nominal operation and maintaining the comfortable temperature in Zone 1 at the desired value.

\section{References}

Afram, A. and Janabi-Sharifi, F. (2014). Theory and applications of HVAC control systems-A review of model predictive control (MPC), Building and Environment 72: 343-355.

Afram, A. and Janabi-Sharifi, F. (2017). Supervisory model predictive controller (MPC) for residential HVAC systems: Implementation and experimentation on archetype sustainable house in Toronto, Energy and Buildings 154: 268-282.

Aftab, M., Chen, C., Chau, C.-K. and Rahwan, T. (2017). Automatic HVAC control with real-time occupancy recognition and simulation-guided model predictive control in low-cost embedded system, Energy and Buildings 154: 141-156.

Bai, J. and Zhang, X. (2007). A new adaptive (PI) controller and its application in (HVAC) systems, Energy Conversion and Management 48(4): 1043-1054.

Bengea, S.C., Li, P., Sarkar, S., Vichik, S., Adetola, V., Kang, K., Lovett, T., Leonardi, F. and Kelman, A.D. (2015).
Fault-tolerant optimal control of a building (HVAC) system, Science and Technology for the Built Environment 21(6): 734-751.

Bouchama, Z., Essounbouli, N., Harmas, M., Hamzaoui, A. and Saoudi, K. (2016). Reaching phase free adaptive fuzzy synergetic power system stabilizer, International Journal of Electrical Power \& Energy Systems 77: 43-49.

Chabir, K., Sauter, D., Gayed, M.K.B. and Abdelkrim, M.N. (2008). Design of an adaptive Kalman filter for fault detection of networked control systems, 16th Mediterranean Conference on Control and Automation (MED), Ajaccio, France, pp. 1124-1129.

Chabir, K., Sid, M.A. and Sauter, D. (2014). Fault diagnosis in a networked control system under communication constraints: A quadrotor application, International Journal of Applied Mathematics and Computer Science 24(4): 809-820, DOI: 10.2478/amcs-2014-0060.

Chen, J. and Patton, R.J. (2012). Robust Model-based Fault Diagnosis for Dynamic Systems, Springer Science \& Business Media, Norwell, MA.

Chinde, V., Kosaraju, K., Kelkar, A., Pasumarthy, R., Sarkar, S. and Singh, N. (2017). A passivity-based power-shaping control of building HVAC systems, Journal of Dynamic Systems, Measurement, and Control 139(11): 111007.

Darure, T., Yamé, J.-J. and Hamelin, F. (2016). Model-based fault-tolerant control of VAV damper lock-in place failure in a multizone building, 14th International Conference on Control, Automation, Robotics and Vision (ICARCV), Phuket, Tailand, pp. 1-6.

Du, Z., Fan, B., Chi, J. and Jin, X. (2014). Sensor fault detection and its efficiency analysis in air handling unit using the combined neural networks, Energy and Buildings 72: $157-166$.

Jafarov, E.M. (2005). Design modification of sliding mode observers for uncertain MIMO systems without and with time-delay, Asian Journal of Control 7(4): 380-392.

Jiang, Z. and Dougal, R.A. (2004). Synergetic control of power converters for pulse current charging of advanced batteries from a fuel cell power source, IEEE Transactions on Power Electronics 19(4): 1140-1150.

Kim, W. and Katipamula, S. (2018). A review of fault detection and diagnostics methods for building systems, Science and Technology for the Built Environment 24(1): 3-21.

Kolesnikov, A., Veselov, G. and Kolesnikov, A. (2000). Modern Applied Control Theory: Synergetic Approach in Control Theory, TRTU, Moscow/Taganrog, pp. 4477-4479.

Kuz'menko, A., Kolesnikov, A. and Kolesnitchenko, D. (2015). Novel robust control of hydrogenerator: The synergetic approach, IFAC-PapersOnLine 48(11): 451-456.

Medjbeur, L., Harmas, M., Benaggoune, S. and Zehar, K. (2018). An adaptive fuzzy $h$ synergetic approach to robust control, Journal of Dynamic Systems, Measurement, and Control 140(1): 011008.

Nusawardhana, A., Zak, S. and Crossley, W. (2007). Nonlinear synergetic optimal controllers, Journal of Guidance, Control, and Dynamics 30(4): 1134-1147. 
Patton, R.J., Frank, P.M. and Clarke, R.N. (1989). Fault Diagnosis in Dynamic Systems: Theory and Application, Prentice-Hall, Upper Saddle River, NJ.

Qi, X., Theilliol, D., He, Y. and Han, J. (2017). An active fault-tolerant control framework against actuator stuck failures under input saturations, International Journal of Applied Mathematics and Computer Science 27(4): 749-761, DOI: 10.1515/amcs-2017-0052.

Sauter, D. and Hamelin, F. (1999). Frequency-domain optimization for robust fault detection and isolation in dynamic systems, IEEE Transactions on Automatic Control 44(4): 878-882.

Sauter, D., Yamé, J., Aubrun, C. and Hamelin, F. (2015). Design of fault isolation filter for control reconfiguration: Application to energy efficiency control in buildings, $23 \mathrm{rd}$ Mediterranean Conference on Control and Automation (MED), Torremolinos, Spain, pp. 197-202.

Seybold, L., Witczak, M., Majdzik, P. and Stetter, R. (2015). Towards robust predictive fault-tolerant control for a battery assembly system, International Journal of Applied Mathematics and Computer Science 25(4): 849-862, DOI: 10.1515/amcs-2015-0061.

Slotine, J.-J.E. and Li, W. (1991). Applied Nonlinear Control, Prentice Hall, Englewood Cliffs, NJ.

Utkin, V.I. (2013). Sliding Modes in Control and Optimization, Springer Science \& Business Media.

Veselić, B., Draženović, B. and Milosavljević, Č. (2014). Sliding manifold design for linear systems with unmatched disturbances, Journal of the Franklin Institute 351: 1920-1938.

Yu, Y., Woradechjumroen, D. and Yu, D. (2014). A review of fault detection and diagnosis methodologies on air-handling units, Energy and Buildings 82: 550-562.

Zhu, W., Zheng, Y., Dai, J. and Zhou, J. (2017). Design of integrated synergetic controller for the excitation and governing system of hydraulic generator unit, Engineering Applications of Artificial Intelligence 58: 79-87.
Radhia Ettouil was born in 1991. She received her engineer's degree in industrial and automatic computing in 2014 from the National Institute of Applied Science and Technology of Tunis (INSAT) and her PhD in electrical engineering at the Laboratory for Modeling, Analysis and Control Systems (MACS), University of Gabes. Her research interests are in fault tolerant control of nonlinear systems.

Karim Chabir received his BS degree in electrical and control engineering in 2003 from the Higher School of Sciences and Technology of Tunis (ESSTT). He received his MSc in automatic and intelligent techniques in 2006 from the National Engineering School of Gabes (Tunisia) and $\mathrm{ahD}$ in automatic control from Henri Poincare University (France) and the University of Gabes in 2011. His research works have been carried out at the Research Center for Automatic Control of Nancy (CRAN) and at the Research Unit for Modeling, Analysis and Control Systems of the National Engineering School of Gabes. He has once been a member of the dependability and system diagnosis group (SURFDIAG). He is now an assistant professor at the National Engineering School of Gabes (ENIG). His current research interests are focused on model-based fault diagnosis and fault-tolerant control, with emphasis on networked control systems.

Dominique Sauter received his $\mathrm{PhD}$ degree in 1991 from Nancy University, France. Since 1993 he has been a full professor at that university, where he teaches automatic control. He is a member of the Research Center in Automatic Control of Nancy (CRAN), associated with the French National Center for Scientific Research (CNRS). His current research interests are focused on model-based fault diagnosis and fault tolerant control, with emphasis on networked control systems.

Momamed Naceur Abelkrim received his BSc in technical sciences in 1980, his MSc in control in 1981 from the ENSET School of Tunis (Tunisia), his PhD in control in 1985, and his DSc in electrical engineering in 2003 from the ENIT School of Tunis. Since 2003, he has been a professor at the Electrical Engineering Department (control) of the National Engineering School of Gabes (Tunisia), and he is the head of the Modelling, Analysis and Control Systems (MACS) Laboratory.

Received: 19 January 2018

Revised: 6 September 2018 Accepted: 22 January 2019 